\title{
Nachruf
}

Franz Mesch und Jürgen Beyerer*

\section{In memoriam Professor Dr.-Ing. Fernando Puente León}

https://doi.org/10.1515/teme-2020-0067

Eingang 27. August 2020; angenommen 1. September 2020

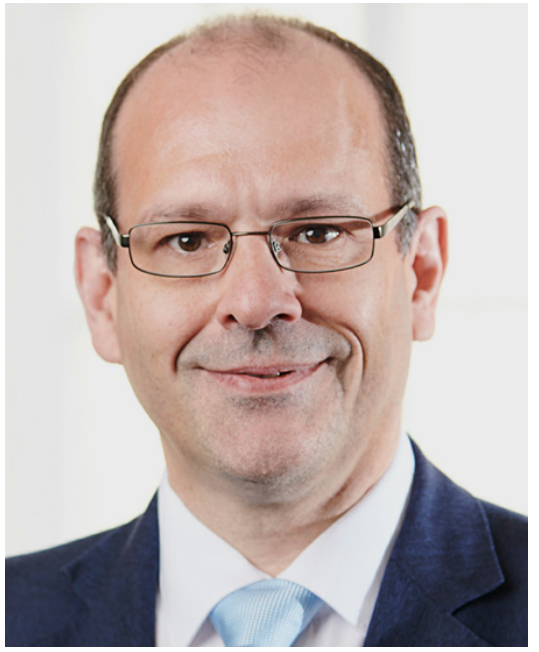

Professor Dr.-Ing. Fernando Puente León†

Gleich drei wissenschaftliche Communities haben ein hervorragendes Mitglied und einen besonderen Menschen verloren: die Automatisierungstechnik, Maschinensehen und Bildverarbeitung sowie die Mess- und Regelungstechnik. Professor Dr.-Ing. Fernando Puente León starb völlig unerwartet und viel zu früh am 1. Juli 2020 im Alter von nur 51 Jahren.

Fernando Puente León wurde am 2. Mai 1969 in Valencia geboren. Sein Vater bekleidete das Amt des Bürgermeisters und andere öffentliche Ämter, die Muttersprachen waren Spanisch und Katalonisch. Er besuchte dort deutsche Schulen und sprach schließlich völlig akzentfrei Deutsch. Danach studierte er an der damaligen Universität Karlsruhe (TH) Elektrotechnik, wurde wissenschaftlicher Angestellter am Institut für Mess- und Regelungstechnik und promovierte 1999 zum Doktor-Ingenieur. Nach einem

*Korrespondenzautor: Jürgen Beyerer, Fraunhofer IOSB, Fraunhoferstr. 1, 76137 Karlsruhe, Germany, E-Mail: juergen.beyerer@iosb.fraunhofer.de

Franz Mesch, KIT, Karlsruhe, Germany
Jahr bei einer spanischen Firma in seiner Heimatstadt war er an der TU München von 2003 bis 2008 Universitätsprofessor und Leiter des Fachgebietes Verteilte Mess-Systeme. Seit 2008 war er als Ordinarius am KIT Karlsruhe Leiter des Instituts für industrielle Informationstechnik IIIT und seit Oktober 2018 führte er die KIT-Fakultät für Elektrotechnik und Informationstechnik als Dekan.

Sein berufliches Engagement war bemerkenswert vielfältig. Neben verschiedenen Ämtern in deutschen und amerikanischen Fachgremien war er gemeinsam mit Prof. Bernhard Zagar Herausgeber der renommierten Fachzeitschrift „tm - Technisches Messen“. Er veranstaltete zahlreiche nationale und internationale Fachkonferenzen und war seit 2008 wissenschaftlicher Berater des FraunhoferInstituts für Optronik, Systemtechnik und Bildauswertung IOSB. Er war treuer Teilnehmer des Regelungstechnischen Kolloquiums in Boppard und er war überaus engagiert im Arbeitskreis der Hochschullehrer für Messtechnik AHMT e.V., bei dem er ab 2011 im Vorstand und von 2015 bis 2016 Vorstandsvorsitzender war.

Halbe Sachen waren ihm fremd. Wenn Prof. Puente León etwas anpackte, dann ganz und gar.

Mit Fernando verbindet uns eine lange gemeinsame Zeit, in der er wissenschaftliche Hilfskraft, Diplomand, Doktorand, Oberassistent und Kollege war. Eine Zeit voller Kreativität, ambitionierter gemeinsamer Projekte und eine Zeit intensiver Verbundenheit und der Freundschaft. Fernando glänzte nicht nur in der Wissenschaft. Alles was er anpackte, tat er mit großem Enthusiasmus. Alle Werkzeuge, die er verwendete, beherrschte er virtuos - und das galt nicht nur beruflich, sondern auch für seine Hobbies $\mathrm{Mu}$ sik und Fotografie. Als Assistent war er, sogar für die deutschen Kolleginnen und Kollegen, der Experte, wenn es um die Feinheiten der deutschen Sprache und ihrer Grammatik ging.

Mit Professor Dr.-Ing. Fernando Puente León verlieren wir eine herausragende Persönlichkeit, einen anerkannten Wissenschaftler und Hochschullehrer, einen hochgeschätzten Kollegen, einen langjährigen Weggefährten und einen guten Freund. Wir werden ihn schmerzlich vermissen.

Seiner Familie und insbesondere seiner Frau und seinen Kindern gilt unser tief empfundenes Mitgefühl. 
Mit Herrn Professor Dr.-Ing. Fernando Puente León verliert der De Gruyter Verlag einen seiner wichtigsten Lehrbuchautoren und Zeitschriftenherausgeber. Mit seinen Büchern „Signale und Systeme“ und „Ereignisdiskrete Systeme" gestaltete er ausgezeichnete Lehrbücher, die die Anforderungen sowohl der Studierenden wie auch seiner Kollegen der Lehre voll erfüllten. Als langjähriges Beiratsmitglied der Zeitschrift ,at - Automatisierungstechnik“ und vor allem als Herausgeber der Zeitschrift „tm - Technisches Messen“ setzte er prägende Qualitätsstandards, die er höchst sorgfältig sicherstellte.

Für die Mitarbeiter und Mitarbeiterinnen des Verlages war er ein verlässlicher Ratgeber, der ihnen mit seinem Wissen und seiner Klugheit jederzeit hilfreich zur Seite stand. So war er ein wertvoller und unermüdlicher Partner für die Weiterentwicklung des Buchprogramms sowie der Zeitschrift „tm - Technisches Messen“.

Über alle dem stand jedoch der Mensch, der mit seinem Enthusiasmus und seinem Optimismus die Zusammenarbeit zu einer reinen Freude machte. Wir fühlen uns geehrt, ihn kennengelernt zu haben und spüren die Lücke, die sein Fortgang hinterlässt schon jetzt schmerzlich. Wir werden ihn alle sehr vermissen.

Unser tiefstes Mitgefühl gilt seiner Familie, insbesondere seiner Frau und seinen Kindern.
Wolfgang Böttner, Claudia Hill, Ulrike Kitzing, Leonardo Milla und Angelika Sperlich für den Walter De Gruyter Verlag und Bernhard Zagar als Co-Herausgeber der Zeitschrift tm - Technisches Messen.

\section{Autoreninformationen}

\section{Franz Mesch}

KIT, Karlsruhe, Germany

mesch@kit.edu

Prof. em. Dr.-Ing. Franz Mesch Ehemals Leiter des Instituts für Messund Regelungstechnik, Universität Karlsruhe (TH), heute KIT.

\section{Jürgen Beyerer}

Fraunhofer IOSB, Fraunhoferstr. 1, 76137 Karlsruhe, Germany juergen.beyerer@iosb.fraunhofer.de

Prof. Dr.-Ing. habil. Jürgen Beyerer Direktor des Fraunhofer-Instituts für Optronik, Systemtechnik und Bildauswertung IOSB und Lehrstuhl für Interaktive Echtzeitsysteme IES, Institut für Anthropomatik und Robotik am KIT. 